\title{
An Overview on Osteoporosis and Herbal Plants Used for Treatment
}

\author{
Swarnima Pandey ${ }^{1 *}$ and Sushant Kumar ${ }^{2}$ \\ ${ }^{1}$ Department of Pharmacy, Hygia College of Pharmacy, Affiliated to Dr. APJ AKTU, \\ Lucknow, India \\ ${ }^{2}$ Department of Pharmacy, Uttar Pradesh University of Medical Sciences, Saifai, \\ Etwah, India \\ *Corresponding Author: Swarnima Pandey, Department of Pharmacy, Hygia \\ College of Pharmacy, Affiliated to Dr. APJ AKTU, Lucknow, India
}

DOI: $10.31080 /$ ASPS.2022.06.0859
Received: January 31, 2022

Published: February 23, 2022

(C) All rights are reserved by Swarnima

Pandey and Sushant Kumar.

\begin{abstract}
The development of osteoporosis begins when the bone becomes porous and weaker, due to which the risk of fractures increases, especially in the hips, spinal vertebrae, and some peripheral joints.

In this review article, we have compiled the causes, risk factors, and various herbal plants which can be used for the treatment of osteoporosis.
\end{abstract}

Keywords: Osteoporosis; Bone Matrix; Herbal Plants

\section{Introduction}

Osteoporosis is a bone disease which is characterized by low bone density, deterioration of bone, which ultimately leads to the risk of increasing bone fragility and hence bone fracture. Globally this disease is a major health risk covering a large area of population which is affected by this Osteoporosis is a bone disease which is characterized by the low bone density, deterioration of bone, which ultimately leads to the risk of increasing bone fragility and hence bone fracture. Globally this disease is a major health risk covering a large area of population which is affected by this [1]. It is a health issue in older population with an approximate prevalence of $14-16 \%$ in women aged 50 and older [2]. The most standard cause of this disease is hypogonadism which generally notion to be an aged adjusted symptom [3]. There is a lifetime risk of typical osteoporosis fracture which increases to $30-40 \%$ in women older than 50 [4]. The likelihood of fracture increases by 1.5-9.5 times in the future. Fragility fractures depends on patients' age, number and site of previous fractures also it can greatly impact one's quality of life, often resulting in impaired mobility and loss of independence in future [5]. Fragility fractures are however can be corrected by treating osteoporosis [7]. Every year, more than 2 million fractures occur in the United States. This disease is still underdiagnosed, and treatment options include adequate calcium and vitamin D levels, exercise to improve balance and prevent falls, and pharmaceutical therapy to reduce fracture risk. Rests are also inadequate [7] and evidence-based guidelines have emphasised the need of suitable non-medical and medical therapies for osteoporosis patients [8], but there is still a widespread and persistent preventative care gap for osteoporosis around the world [9]. The relationship between bone mineral density and bone mass at maturity (peak bone mass) and subsequent bone loss in this patient has been established. Bone remodels throughout one's life, with the rate of remodelling increasing as one gets older. In elderly adults, the pace of reabsorption exceeds the rate of creation, resulting in very thin bones, or osteoporosis. Bone remodelling occurs at different locations throughout the skeleton and proceeds in a well-ordered manner, with bone reabsorption always followed by bone production, a phenomenon known as coupling. In cortical and cancellous bone, the sequence is comparable. It takes up to eight 
months to complete the process of bone resorption, followed by the synthesis of bone matrix and its subsequent mineralization. If the processes of bone resorption and formation are not balanced, bone resorption and formation will occur.

\section{Definition of osteoporosis}

Osteoporosis is largely conceptual, making it difficult to apply to particular patients. Osteoporosis is defined as a bone mineral density (T score) that is $2.5 \mathrm{SD}$ below the mean peak value in young people, according to a WHO working committee [11]. This definition has limits in clinical practise and can be used as an entrance criterion for a clinical trial or as a tool to study the epidemiology of osteoporosis. It elevates the hazard element for a fracture to the status of a diagnostic criterion, does not specify the method or site for measuring bone mineral density, ignores the importance of other determinants of bone strength, and also ignores the increased risk of fracture associated with a certain level of bone mineral density [12]. The annual cost of understanding for osteoporosisrelated fractures in the United States is comparable to or exceeds the cost of understanding for myocardial infarction, breast cancer, and/or cerebrovascular accident in the United States [13]. There are specific risk factors that differ from person to person that are linked to the development of osteoporosis and increase the risk of getting the condition. These factors are classified into two categories: modifiable and non-modifiable factors. Calcium and vitamin D intake, anorexia nervosa, alcohol consumption, cigarette smoking, pharmaceutical use, and sex hormones are all modifiable factors. Age, sex, race, body size, family history, and so on are non-modifiable characteristics.

\section{Pathogenesis of osteoporosis}

Osteoblasts and osteocytes (bone-forming cells), osteoclasts (bone-resorbing cells), and osteoid cells make up bone (bone matrix). When the balance between bone resorption and deposition swings toward excessive resorption, bone loss occurs as a precursor to osteoporosis [14]. Osteoporosis is a multifaceted illness with complicated pathophysiology that can be triggered by genetic, endocrine, or dietary factors.

Several hormones have been proven to aid in bone growth, the most important of which are parathyroid hormone (PTH), oestrogen, calcitonin, and vitamin D.PTH improves calcium absorption in the kidneys, gut, and bones.
Furthermore, PTH can promote osteoclast activity, resulting in increased bone resorption.

Calcitonin plays a vital role in calcium prevention by inhibiting calcium absorption both directly and indirectly. Calcitonin reversibly blocks I osteoclasts role by binding to its binding to its receptor. Estrogen decreases the irate of bone remolding and increases osteoclast apoptosis through two receptors also including estrogen receptor $i \alpha \mathrm{i}(\mathrm{ER} \alpha)$ and estrogen receptor $\mathrm{i} \beta \mathrm{i}(\mathrm{ER} \beta)$.Due to its relationship with increased bone resorption and defective bone formation, estrogen insufficiency plays a censorious role in the aetiology of osteoporosis [16].

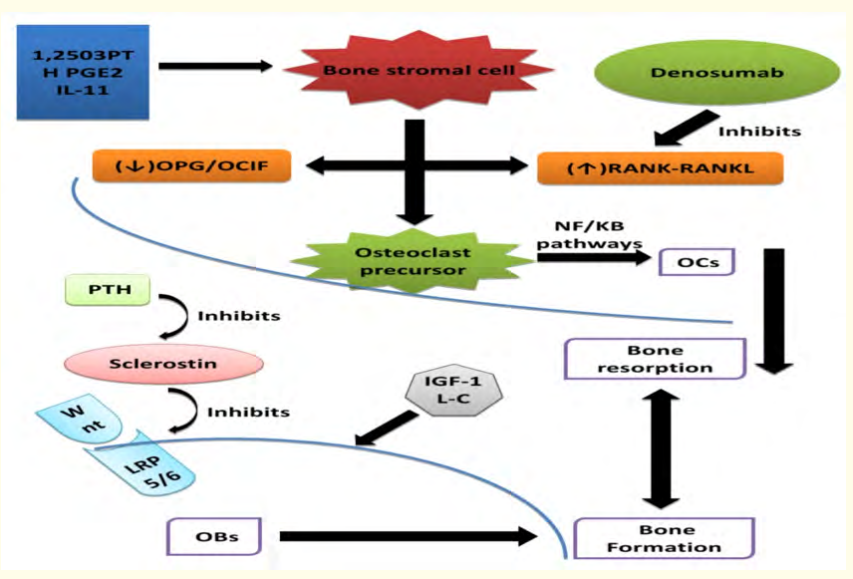

Figure 1: A brief schematic view of pathophysiology of osteoporosis and influence of some drugs ion bone health.

PTH: Parathyroid Hormone; PGE2: Prostaglandin E2; IL-11: Interleukin-11; OPG: Osteoprotegerin; OCIF:

Osteoclastogenesis Inhibitory Factor; RANKL: Receptor Activator of Nuclear Factor $\kappa B$ Ligand; NF- $\kappa B$ : Nuclear Factor iкB; JNK: c-Jun N-terminal Kinases; OSc: Osteoclasts; Wnt: Wingless and Int Proteins; LRP5/6: LDL-Receptor Related Protein i5/6; iIGF-1: Insulin Like Growth Factor-1; L-C: L-carnitine; OBs: Osteoblasts.

\section{Literature Review}

\section{Fractures caused by osteoporosis}

One in every three women and one in every five men over the age of fifty is at risk of an osteoporosis fracture. In fact, it is be- 
lieved that an osteoporosis fracture occurs every 3 seconds. The hip, wrist, and spine are the most prevalent sites for osteoporosisrelated fractures. In both women and men, the likelihood of these fractures occurring, particularly at the hip and spine, increases with age [17].

\section{Osteoporosis classification}

When analysing the factors that affect bone metabolism, osteoporosis is divided into two groups:

- Primary osteoporosis is the most common type of osteoporosis.

- $\quad$ Secondary osteoporosis.

Primary osteoporosis and secondary osteoporosis are two types of osteoporosis, based on the factors that influence bone metabolism.

There are three forms of primary osteoporosis.

Postmenopausal osteoporosis - occurs when a woman's oestrogen levels drop after menopause, causing bone loss. It is defined by rapid loss of trabecular bone mass with perforation, while cortical bone is partially spared. This loss is also vulnerable for fragility fractures due to load bearing, particularly in the vertebrae and distal radius. It also has a high bone turnover rate, bone marrow growth, and a high frequency of increased endothelial resorption, as well as periosteal bone formation inhibition [18].

Osteoporosis in children and teenagers is common.

Juvenile osteoporosis is a term that is widely used to describe a type of osteoporosis that occurs in children and adults. This disease is mostly caused by genetic abnormalities that can lead to quantitative or qualitative changes in the connective tissue component of bone, as well as altered osteoblastic activity with trabecular bone involvement. It can also be caused by chronic drug administration, such as anti-epileptics and glucocorticoids, or by leukaemia, extended immobility, or chronic inflammatory illnesses. It is known as juvenile idiopathic osteoporosis when it is impossible to recognise possible causes of it of bone loss and fragility fracture.

\section{Osteoporosis in men and seniors}

Secondary osteoporosis refers to patients who have a causal factor or disease process that can be seen [19]. Due to the unique diag- nostic and therapeutic implications, it's important to pay attention. Here are some warning signs for some of the most common forms of secondary osteoporosis management.

Chronic glucocorticoid exposure, due to increased endogenous synthesis (Cushing's syndrome), and to exogenous intake (treatment of inflammatory or autoimmune disease), is an important cause of osteoporosis and By reducing osteoblast proliferation and differentiation and boosting osteoblast and osteocyte apoptosis, glucocorticoids stimulate resorption and decrease bone formation. Fragility fractures occur in between 30 and 50\% of patients in the first 5 years of chronic glucocorticoid therapy and their chances is If other risk factors, such as old age, previous fractures, and in women, menopause, are present, further rise [20].

\section{Organ transplant osteoporosis}

The appraised prevalence of fragility fractures is around 1015 percent in patients waiting for solid organ transplant (kidney, heart, liver, and lungs), due the pessimistic effects. The percentage of patients with osteoporosis rises completely after transplant. After transplant, the percentage of vertebral fractures due to bone fragility reaches a park and also affects $30-40 \%$ of patients [18].

\section{Drug osteoporosis}

Epidemiological and retrospective research show that a variety of medicines are linked to osteoporosis and fragility fractures. In addition to steroid therapy, aromatase inhibitors and GnRH have now been linked to an increased risk of fragility fractures. The use of proton pump inhibitors has been linked to a considerably increased incidence of vertebral and hip fractures.

\section{Causes and risk factors}

Doctors have diagnosed a few danger elements for osteoporosis. Some are adaptable, however it's far not possible to disregard others. The frame regularly absorbs antique bone tissue and produces new bone to keep bone density, strength, and structural integrity [22]. Bone density peaks whilst someone is of their 20s, and it starts to weaken at approximately 35 years of age. It can have an effect on each men and females, however it's far maximum possibly to appear in girls after menopause due to the surprising lessen in estrogen. Estrogen commonly protects girls towards osteoporosis [23]. 


\section{Etiology and pathogenesis of osteoporosis}

Bone strength displays the mixing of 2 important features: Bone Density and every other is Bone Quality [24]. Many elements offer to the possibility of osteoporosis fractures, all of which be taken under consideration withinside the assessment of fracture danger in patients [25].

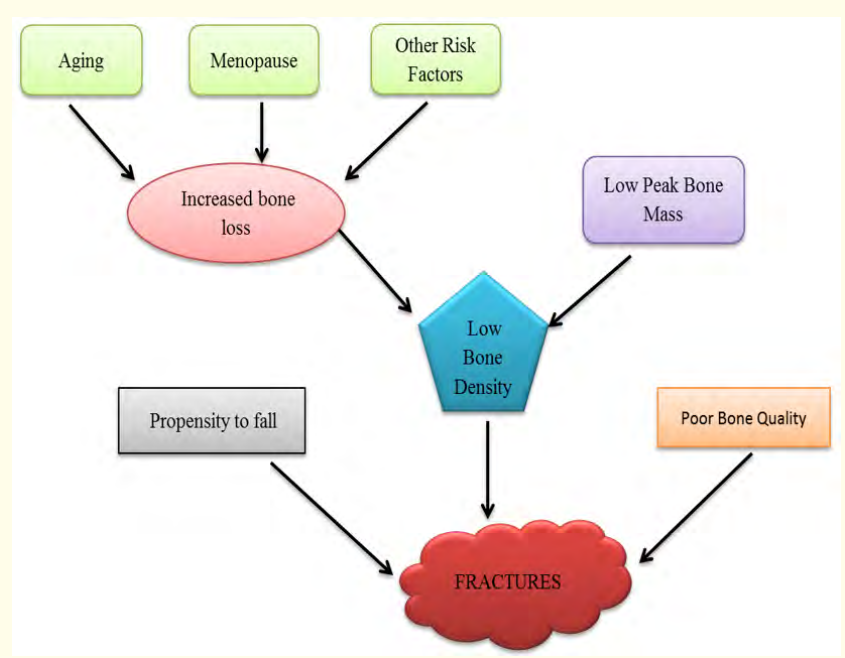

Figure 2: Schematic representation of etiology and pathogenesis of osteoporosis.

\section{Anti-osteoporotic plant}

Natural merchandise for the supervision of osteoporosis is on the whole phytoestrogens which encompass isoflavones, lignins, flavonoids and coumestans that percentage structural and purposeful similarities' with evidently taking place or artificial estrogens. Phytoestrogens show off like results at diverse reproductive and non-reproductive tissues [28]. To save you all below physical, intellectual and economic struggling with the aid of using patients,
Sign I and I symptoms of osteoporosis

Early withinside the direction of the disease, osteoporosis might also additionally reason no symptoms. Later, it could reason decreasing top or continual ache withinside the bones or muscles, mainly low returned ache or neck ache. Later withinside the direction of the disease, acute pains might also additionally come ion instantly. The ache won't diffuse (unfold to different areas), it could be made worse via way of means of interest that places weight ion the area, can be followed via way of means of tenderness and commonly began out to subside in a single week. Pain might also additionally stay extra than three months. People with osteoporosis won't even don't forget a fall or different trauma that could reason a damaged bone, inclusive of withinside the backbone or foot. Spinal compression fractures might also additionally bring about lack of top with a stooped (known as a dowager's hump). Fractures at different sites, generally the hip or bones of the wrist, commonly end result from a fall [26].

\section{Treatment}

- Maintain healthy bone mineral density and Bone mass

- Slow or prevent the development of osteoporosis

- Prevent fractures

- Maximize the person's ability to continue with their daily

- Reduce pain [27].

there may be an excessive significance for the higher opportunity healing control particularly from herbal sources which might be notion to be more healthy and more secure for the remedy of osteoporosis [29]. Many medicinal flora were used to save you and deal with osteoporosis in diverse countries. These herbal drug treatments acquired from flora additionally have little facet impact and are greater suitable for long-time period use than synthesized drugs.

\begin{tabular}{|l|c|c|c|c|}
\hline Scientific name & Family & Plants parts used & $\begin{array}{c}\text { Reported relevant ethno } \\
\text { medical use }\end{array}$ & Chemical constituents \\
\hline $\begin{array}{l}\text { Achyranthes } \\
\text { bidentata Blume }\end{array}$ & Amaranthaceae & Root & Bone related diseases & $\begin{array}{c}\text { Oleanolic acid glucosides, } \\
\text { ecdysone and allantion }\end{array}$ \\
\hline Uminum cyminum L. & Apiaceae & Fruit & Toothache, diarrhea, epilepsy & $\begin{array}{c}\text { B-sitosterol, stigmasterol, } \\
\text { luteolin and apiigenin }\end{array}$ \\
\hline Berberis aristata DC & Berberidaceae & Stem bark & $\begin{array}{c}\text { Menopausal disorders, } \\
\text { osteoporosis }\end{array}$ & $\begin{array}{c}\text { Berberine chloride, } \\
\text { palmatine chloride, } \\
\text { canadine }\end{array}$ \\
\hline
\end{tabular}




\begin{tabular}{|l|c|c|c|c|}
\hline Carthamus tinctorius L. & Compositae & Seed & Rheumatism and chronic nephritis & $\begin{array}{c}\text { Lignas, flavones and } \\
\text { serotonins }\end{array}$ \\
\hline Silybum williamsii Hance & Compositae & Seed & Liver disease & $\begin{array}{c}\text { Silibinin, isosilibinin, } \\
\text { silydianin }\end{array}$ \\
\hline $\begin{array}{l}\text { Vaccinium angustifolium } \\
\text { Aiton }\end{array}$ & Ericaceae & Fruit & Cardiovascular disease & $\begin{array}{c}\text { Phenolic acids, flavonoids, } \\
\text { lignans }\end{array}$ \\
\hline $\begin{array}{l}\text { Abelmoschus manihot } \\
\text { (L) Medik }\end{array}$ & Malvaceae & Leaf & Calcium \\
\hline
\end{tabular}

Table 1: Antiosteoporosis medicinal plants W chemical constituents and therapeutic I applications.

\section{Epimedium plants}

Epimedium (Berberidaceae) is a low-growing, deciduous, perennial plant. The leaves of E. brevicomum Maxim, E. sagittatum (Sieb.et Zucc) Maxim, E. pubescens Maxim, E. koreanum Nakai, E. wushanense. T.S. Ying have lengthy been used it save you and deal with osteoporosis and different menopause sicknesses in China. those are the maximum frequently used herb pills in antiosteoporotic Chinese conventional remedy formula [3]. Flavonoids including carin, epimedin B and epimedin C are the primary antiosteoporotic constituents, which inhibit bone resorption, stimulate bone formation, suppress urinary calcium excretion and as a consequence save you osteoporosis without hyperplastic outcomes ion the uterus withinside the ovariectomized (OVX) rat model.

\section{Glycine $\max \mathrm{L}$}

Glycine max L. (Fabaceae) first of all grows withinside the southwest of Asia and at present, it's far widely planted in warm zone. its seed additionally referred to as soybean, is likewise a fitness complement and consists of enough of nutritional substances, along with proteins and flavonoids such as daidzein, genistein and biochanin A. The soy flavonoids which are structurally and functionally linked to i17-beta-estradiol have sturdy outcomes on bone metabolism in postmenopausal ladies and feature a component in prevention and remedy of postmenopausal osteoporosis.

\section{Psoralea corylifolia L}

Psoralea corylifolia $\mathrm{L}$. belongs to the family Leguminosae, and this fruit is one of the herbs regularly used in prescribed procedures to treat fractures, bones and joint diseases. New research is P. It suggests that corylifolia has a strong estrogenic effect and its fruits may be an effective treatment for fractures, osteomalacia, and osteoporosis. Excerpt from P Fruits of Psoralea corylla not only significantly increase serum inorganic phosphorus levels, but can also clearly develop bone mineralization in rats. Both its fruit and seed extracts and the two isoflavones (colyline and babatin) isolated from this plant can stimulate bone development and exhibit anti-osteoporotic activity.

\section{Salvia miltiorrhiza Bunge}

Salvia miltiorrhiza Bunge (Labiatae) is a conventional Chinese medicine, broadly used in clinical exercise for the prevention and treatment of cardio-cerebral vascular diseases. Pharmacological testing showed that $S$. miltiorrhiza has anticoagulant, vasodilatory, increased blood flow, anti-inflammatory, free radical scavenging, mitochondrial protective activities. Phytochemical studies divulged various groups of compounds from S. miltiorrhiza Bunge extract, the main constituents of which include tanshinones (tanshinone I, tanshinone IIA, cryptotanshinone, i15,16-dihydrotanshinone I) and phenolics (protocatechuic aldehyde, salvianolic acid A and salvianolic acid B).

\section{Drynaria fortunei (Kunze) J. Sm}

Drynaria Fortunei (Kunze) J of the fern family. Sm. Rhizome has a long healing experience in East Asia and is used to treat gynaecological disorders such as inflammation, arteriosclerosis, hyperlipidaemia and osteoporosis. Traditional Chinese and Korean remedies for treating osteoporosis usually include Drynaria fortunei rhizomes. Recent studies have shown that Drynaria fortunei has a therapeutic effect on osteoporosis and fractures in ovariectomized rat models and improves bone formation.

\section{Pueraria lobata and P. mirifca}

Pueraria lobata (Wild) Ohwi is an untouched vine of the leguminous family. Its roots are one of the earliest and very important herbs used in Chinese herbs for a variety of medicinal motivations 
and are rich in is of lavonoids such as daidzein and genistein. P. lobata root showed a preventive effect on bone loss by prolonging BMD (bone mineral density) in rats and mice after ovariectomy and orchiectomy without showing estrogenic effects in utero. increase. Puerarin, a natural is of lavonoid contained in P. lobata, significantly increased osteoblast cell viability, ALP activity, and mineral nodule formation by activating the iP13K/Akt pathway.

\section{Conclusion}

In the future, doctors may use stem cell treatment to treat osteoporosis. Researchers discovered in 2016 that injecting a certain type of stem cell into mice cured osteoporosis and bone loss in a way that could benefit humans.

Bone density is thought to be mostly determined by genetic factors, according to scientists. Researchers are looking at which genes are involved in bone growth and loss in the hopes of developing a new osteoporosis treatment.

\section{Bibliography}

1. McCloskey E., et al. "FRAX-based assessment and intervention thresholds-an exploration of thresholds in women aged 50 years and older in the UK". Osteoporosis International 26.8 (2015): 2091-2099.

2. Chen P., et al. "Prevalence of osteoporosis in China: a metaanalysis and systematic review". BMC Public Health 16.1 (2016): 1039.

3. Jia M., et al. "Potential antiosteoporotic agents from plants: a comprehensive review". Evidence-Based Complementary and Alternative Medicine 2012 (2012).

4. Cummings SR., et al. "Risk factors for hip fracture in white women”. New England Journal of Medicine 332.12 (1995): 767773.

5. Drake MT., et al. "Risk factors for low bone mass-related fractures in men: a systematic review and meta-analysis". The Journal of Clinical Endocrinology and Metabolism 97.6 (2012): 1861-1870.

6. Bone HG., et al. "Ten years' experience with alendronate for osteoporosis in postmenopausal women". New England Journal of Medicine 350.12 (2004): 1189-1199.

7. Sözen T., et al. "An overview and management of osteoporosis". European Journal of Rheumatology 4.1 (2017): 46.
8. Runowicz CD., et al. "American cancer society/American society of clinical oncology breast cancer survivorship care guideline". CA: A Cancer Journal for Clinicians 66.1 (2016): 43-73.

9. Lewiecki EM., et al. "Hip fracture trends in the United States, 2002 to 2015". Osteoporosis International 29.3 (2018): 717722.

10. Eastell R. "Treatment of postmenopausal osteoporosis". New England Journal of Medicine 338.11 (1998): 736-746.

11. Kanis JA., et al. "The diagnosis of osteoporosis". Journal of Bone and Mineral Research 9.8 (1994): 1137-1141.

12. Brown JP and Josse RG. "2002 clinical practice guidelines for the diagnosis and management of osteoporosis in Canada". CMAJ 167 (2002): S1-S34.

13. Miller PD. "Underdiagnoses and undertreatment of osteoporosis: the battle to be won". The Journal of Clinical Endocrinology and Metabolism 101.3 (2016): 852-859.

14. Tabatabaei-Malazy 0., et al. "New horizons in treatment of osteoporosis". DARU Journal of Pharmaceutical Sciences 25.1 (2017): 2.

15. Crockett JC., et al. "Bone remodelling at a glance". Journal of Cell Science 124.7 (2011): 991-998.

16. Raisz LG. "Pathogenesis of osteoporosis: concepts, conflicts, and prospects". The Journal of Clinical Investigation 115.12 (2005): 3318-3325.

17. Tuzun S., et al. "Incidence of hip fracture and prevalence of osteoporosis in Turkey: the FRACTURK study". Osteoporosis International 23.3 (2012): 949-955.

18. Nuti R., et al. "Guidelines for the management of osteoporosis and fragility fractures". Internal and Emergency Medicine 14.1 (2019): 85-102.

19. Nancy J. "A study to assess the effectiveness of structured teaching programme on knowledge regarding prevention of osteoporosis among health care personnel working in Rajiv Gandhi Government General Hospital, Chennai: College of Nursing, Madras Medical College, Chennai" (2018).

20. Prescott PT. "Disorders of the adrenal cortex". Medical Management of the Surgical Patient: A Textbook of Perioperative Medicine (2013): 358. 
21. Hadji P., et al. "Management of aromatase inhibitor-associated bone loss in postmenopausal women with breast cancer: practical guidance for prevention and treatment". Annals of Oncology 22.12 (2011): 2546-2555.

22. Otomo-Corgel J. "Osteoporosis and osteopenia: implications for periodontal and implant therapy". Periodontology 2000 59.1 (2012): 111-139.

23. Guggenbuhl P. "Osteoporosis in males and females: Is there really a difference?" Joint Bone Spine 76.6 (2009): 595-601.

24. Prevention O. "Diagnosis, and therapy". NIH Consensus Statement 17.1 (2000): 1-36.

25. Riggs BL and Melton LJ. “Osteoporosis: etiology, diagnosis, and management”. Lippincott Williams and Wilkins (1995).

26. Rao RD and Singrakhia MD. "Painful osteoporotic vertebral fracture: pathogenesis, evaluation, and roles of vertebroplasty and kyphoplasty in its management". JBJS 85.10 (2003): 20102022.

27. Siris ES., et al. "Bone mineral density thresholds for pharmacological intervention to prevent fractures”. Archives of Internal Medicine 164.10 (2004): 1108-1112.

28. Kumar M., et al. "Anti-osteoporotic constituents from Indian medicinal plants". Phytomedicine 17.13 (2010): 993-999.

29. Bateman ED., et al. "Global strategy for asthma management and prevention: GINA executive summary". European Respiratory Journal 31.1 (2008): 143-178.

30. Health Udo and Services H. "Bioanalytical method validation, guidance for industry" (2001).

\section{Assets from publication with us}

- Prompt Acknowledgement after receiving the article

- Thorough Double blinded peer review

- Rapid Publication

- Issue of Publication Certificate

- High visibility of your Published work

Website: www.actascientific.com/

Submit Article: www.actascientific.com/submission.php

Email us: editor@actascientific.com

Contact us: +919182824667

Citation: Swarnima Pandey and Sushant Kumar. "An Overview on Osteoporosis and Herbal Plants Used for Treatment". Acta Scientific Pharmaceutical Sciences 6.3 (2022): 29-35. 